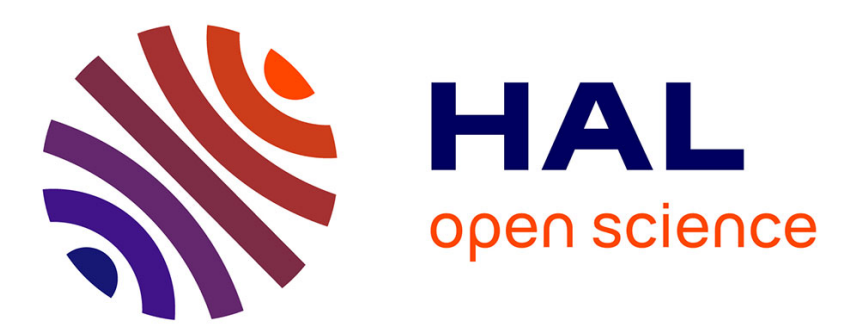

\title{
Adaptive non-uniform sampling of sparse signals for Green Cognitive Radio
}

\author{
Samba Traore, Babar Aziz, Daniel Le Guennec, Yves Louet
}

\section{To cite this version:}

Samba Traore, Babar Aziz, Daniel Le Guennec, Yves Louet. Adaptive non-uniform sampling of sparse signals for Green Cognitive Radio. Computers and Electrical Engineering, 2016, 52, pp.253-265. 10.1016/j.compeleceng.2015.05.002 . hal-01250661

\section{HAL Id: hal-01250661 https://hal.science/hal-01250661}

Submitted on 5 Jan 2016

HAL is a multi-disciplinary open access archive for the deposit and dissemination of scientific research documents, whether they are published or not. The documents may come from teaching and research institutions in France or abroad, or from public or private research centers.
L'archive ouverte pluridisciplinaire HAL, est destinée au dépôt et à la diffusion de documents scientifiques de niveau recherche, publiés ou non, émanant des établissements d'enseignement et de recherche français ou étrangers, des laboratoires publics ou privés. 


\title{
Adaptive non-uniform sampling of sparse signals for Green Cognitive Radio
}

\author{
Samba TRAORÉ, Babar AZIZ, Daniel LE GUENNEC, Yves LOUET \\ IETR/SCEE Centrale-Supélec Campus de Rennes, Avenue de la Boulaie - CS 47601 \\ F-35576 Cesson-Sevigne cedex, France
}

\begin{abstract}
Based on previous results on periodic non-uniform sampling (Multi-Coset) and using the well known Non-Uniform Fourier Transform through Bartlett's method for Power Spectral Density estimation, we propose a new smart sampling scheme named the Dynamic Single Branch Non-uniform Sampler. The idea of our scheme is to reduce the average sampling frequency, the number of samples collected, and consequently the power consumption of the Analog to Digital Converter. In addition to that our proposed method detects the location of the bands in order to adapt the sampling rate. In this paper, through we show simulation results that compared to classical uniform sampler or existing multicoset based samplers, our proposed sampler, in certain conditions, provides superior performance, in terms of sampling rate or energy consumption. It is not constrained by the inflexibility of hardware circuitry and is easily reconfigurable. We also show the effect of the false detection of active bands on the average sampling rate of our new adaptive non-uniform sub-Nyquist sampler scheme.
\end{abstract}

Keywords: Non-Uniform sub-Nyquist sampling, Software Radio, Cognitive radio, Non-Uniform spectrum sensing.

\section{Introduction}

Radio Frequency (RF) allows modulation of narrow band signals with a high carrier frequency. The radio signals of human origin are often sparse. In other words, they are composed of a relatively small number of narrow band 5 transmissions spread across a wide spectral region. A practical description of these signals is the multi-band model where the spectrum of the signal is only composed of several continuous intervals in a wide spectrum. In addition, new wireless applications place high demands on the quality of radio resources such

Email addresses: samba.traore@supelec.fr (Samba TRAORÉ), babar.aziz@supelec.fr (Babar AZIZ), daniel.leguennec@supelec.fr (Daniel LE GUENNEC), Yves.Louet@supelec.fr (Yves LOUET) 
as bandwidth and spectrum. Moreover, the current trends in wireless technol-

ogy have increased the complexity of the receiver, more specifically its Analog to Digital Converter (ADC).According to Shannon-Nyquist theorem, a signal whose spectral support is limited to $-\frac{f_{n y q}}{2}$ and $\frac{f_{n y q}}{2}$ can be perfectly reconstructed by sampling at $f_{n y q}$. To sample a wide band signal with Nyquist rate will require a high sampling rate ADC which consumes a lot of energy. To re-

15 duce the sampling rate, and in turn the energy consumption, several researchers have studied the possibility of sub-Nyquist sampling. In [? ] , a sub-Nyquist sampling method is proposed for sparse multi-band signals, called Modulated Wideband Converter (MWC). MWC consists of several stages and each stage uses a different mixing function followed by a low pass filter and a low uniform 2o sampling rate. This sampling technique shows that perfect reconstruction is possible when the band locations are known. In [? ] [? ], authors have studied the blind case, where the location of the bands is unknown with an inflexible and sub optimal sampler scheme in term of sampling rate. Over the recent years, multi-coset sampling [? ] [? ] [? ] has gained fair popularity and several

25 methods of implementing the multi-coset sampling have been proposed. The most famous architecture is composed of several parallel branches, each with a time shift followed by a uniform sampler operating at a sampling rate lower than the Nyquist rate. Recently, a different approach for implementing multi-coset sampling has been proposed in [? ], which uses uniform samplers operating so at different rates and is known as the Synchronous Mutlirate Sampling. In[? ], a Dual-sampling architecture is presented for multi-coset sampling which is basically a subset of the Synchronous Multi rate Sampling and uses only two uniform samplers. But for optimal reconstruction, all these methods assume that the number of bands and the maximum bandwidth, a band can have, is

35 known. In [? ], authors have defined some requirements that need to be fulfilled for a sampling system to be efficient. These requirements are stated as follows: the sampling rate (average) should be as low as possible, the system has no prior knowledge of the band locations, and the system can be designed with existing devices. In this paper, we propose a blind sampler based on multi40 coset sampling scheme which respects the above stated definition of an efficient sampler. Our scheme estimates the spectral support, using our non-uniform spectrum sensing model proposed in [? ? ], to minimize the average sampling rate, thereby reducing the number of samples as well as energy consumption. We show that our proposed spectrum sensing model provides accurate results 45 using less data samples. Its performance is examined at low rate SNR values with less data samples. In addition, its power consumption is compared with regular ADC when the input signal is very sparse and it is found to produce satisfactory results.

This article is organized as follows. In Section 2 we present the signal model so along with an overview of multi-coset sampling. In Section 3, we present our proposed sampling system and explain all its blocks. Then the non-uniform spectrum sensing model is presented and the functionality of each block is explained. Numerical results are presented in Section 4 followed by a conclusion 
in the end.

\section{2. Background}

\subsection{Multi-band signal Model}

Let $\mathcal{M}(\mathcal{B})$ be the class of continuous real-valued signals with finite energy and band-limited to a subset $\mathcal{B}$.

$$
\mathcal{M}(\mathcal{B})=\left\{x(t) \in L^{2}(\mathbb{R}): \mathbf{X}(f)=0 \forall f \notin \mathcal{B}\right\}
$$

where $\mathcal{B}=\left[-\frac{f_{n y q}}{2}, \frac{f_{n y q}}{2}\right]$ and $\mathbf{X}(f)=\int_{-\infty}^{+\infty} x(t) e^{-j 2 \pi f t} d t$ is the Fourier transform of the signal $x(t)$.

Let $\mathcal{F}$ represent the spectral support of the signal defined by

$$
\mathcal{F}=\bigcup_{i=1}^{N}\left[a_{i}, b_{i}\right]
$$

where $\mathcal{F} \subset \mathcal{B}, a_{i}$ and $b_{i}$ represent, respectively, the lower and upper bound of each bands, and $N$ is the number of bands in $\mathcal{B}$.

\subsection{Multi-Coset Sampling}

Multi-Coset (MC) sampling is a periodic non-uniform sub-nyquist sampling technique which samples a signal $x(t)$ at a lower rate than the Nyquist rate, thereby capturing only the amount of information required for an accurate re-

${ }_{65}$ construction of the signal [? ? ]. In short, the process of MC sampling can be viewed as first sampling the input signal at a uniform rate with period $T$ and then selecting only $p$ non-uniform samples from $L$ equidistant uniform samples. The process is repeated for consecutive segments of $L$ uniform samples such that the $p$ selected samples have a sampling period $L$. The set $\mathcal{C}=\left\{c_{i}\right\}_{i=0}^{p-1}$ 7o specifies the $p$ samples that remain in each segment of length $L$ such that $0 \leq c_{0}<c_{1}<\ldots<c_{p-1} \leq L-1$.

MC sampler is usually implemented by placing $p$ ADCs in parallel as shown in Fig,1. Each ADC operates uniformly at a period $T_{s}=L T$. The $\Delta_{i}=c_{i} T$ represents the time shifts in sampling instants introduced in each branch. It

75 should be noted that a good choice of the sampling pattern $\mathcal{C}$ reduces the margin of error due to spectral aliasing and sensitivity to noise in the reconstruction process [? ]. It is quite evident from Fig.1 that once the sampling parameters (such as $p$ ) are selected, architecture of the MC sampler will remain unchanged irrespective of the input signal characteristics. In other words, once designed,

so the sampler in Fig. 1] cannot be changed because of hardware limitations. If the input signal changes the MC sampler does not adapt, which results in suboptimal sampling of the signal, as will be show later. This motivated us to look for an flexible system which conforms with the spectrum of the input signal. In the next section, we explain the functionality of our new sampling scheme with 85 and show that it is more flexible compared to the MC sampler in Fig, 1. 


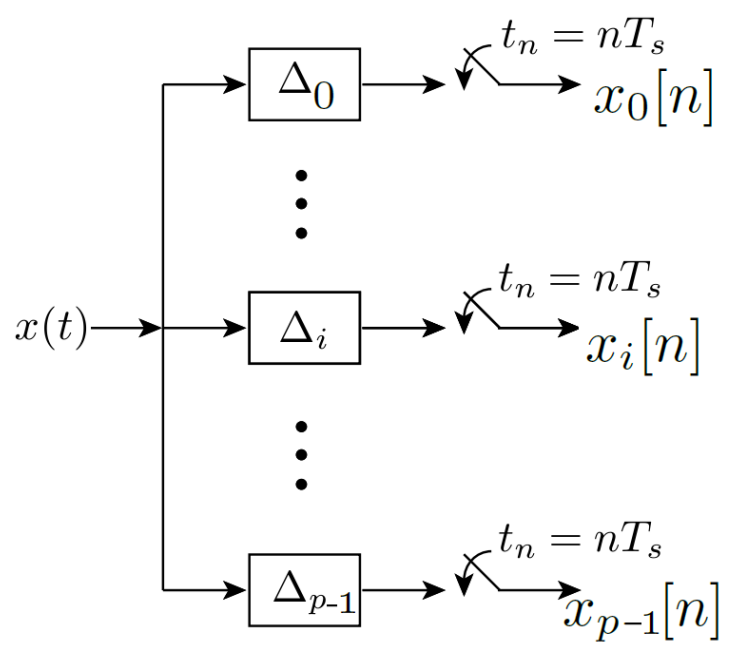

Figure 1: Multi-Coset (MC) sampler implemented as a multi-channel system.

\section{System Model}

With the exponential growth in the means of communications, modifying radio devices easily and cost-effectively has become business critical. Softwaredefined radio (SDR) technology brings the flexibility, cost efficiency and power

9o to drive communications forward, with wide-reaching benefits realized by service providers and product developers through to end users [? ]. One of the main objectives of SDR is to propose new technologies to design radio terminals and wireless infrastructure able to support hardware-independent, multi-service operations and which are remotely reconfigurable. Furthermore, Cognitive radio

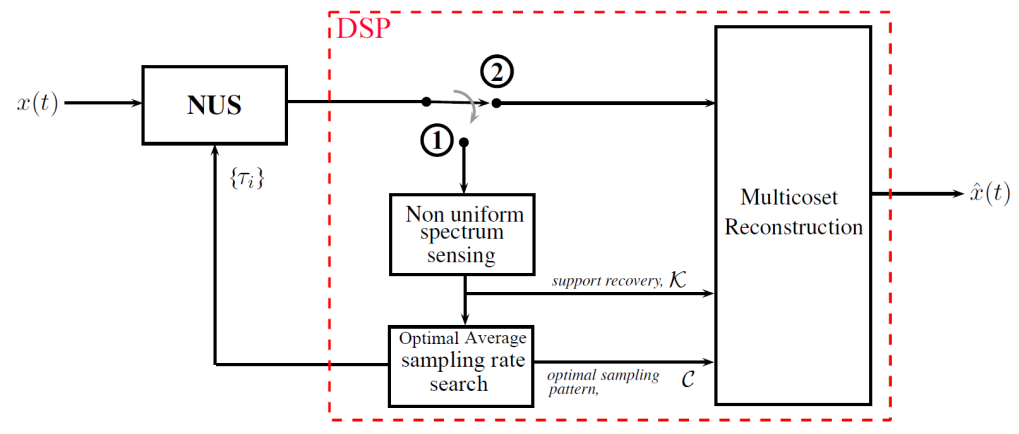

Figure 2: Dynamic Single Branch Non-Uniform Sampler

95 continues to gain popularity as it adapts intelligently to the radio environment, there by dynamically managing the spectrum [? ]. As a result, the spectrum changes continuously and rarely remains constant. Keeping the aspects of Software radio and Cognitive radio in mind, we present in this paper a new sampler, 
that not only adapts to the changes of the input signal but is also remotely reconfigurable and is, therefore, not constrained by the inflexibility of hardwired circuitry. Our proposed non-uniform sampler is shown in Fig. 2. We call it the Dynamic Single Branch Non-Uniform Sampler (DSB-NUS) or simply the DSB sampler [? ]. DSB sampler is based on the principle of MC sampling except that instead of $p$ input branches in case of MC sampling, the DSB sampler 105 has only one. Furthermore, our DSB sampler has an adaptation loop which allows to measure the spectral support of the input signal periodically, and adapt the sampling rate according to the spectrum of the input signal. The regular MC sampling lacks this adaptive and feedback mechanism and is rather a static, hardwired system. The DSB sampler operates in blind mode, without any knowledge of the input signal's spectral support $\mathcal{F}$ and the number of bands $N$, and efficiently reduces the number of samples while ensuring optimal reconstruction with minimum error.

The DSB-NUS operates in two phases:

1. Adaptation phase: When the switch is in position 1, the sampler adapts the sampling parameters according to the spectral support of the input signal $x(t)$ through a feedback loop.

2. Reconstruction Phase: When the switch is in position 2, the DSB-NUS sampler performs Multi-Coset reconstruction.

As this will be shown shortly, the average sampling rate of our system depends

120 on the signal sparsity in the frequency domain. Next we present the operation of each block of the DSB sampler.

\subsection{Non-Uniform Sampler block (NUS)}

In this paper, we design the NUS of the DSB sampler as a reconfigurable Additive Pseudo-Random Sampler (APRS) [? ] in conjunction with MC sampling. Thus, unlike the regular MC sampler with several branches, the input of the DSB-NUS is composed of a single branch non-uniform sampler. The $\mathcal{N}$ sampling instants of the mono-branch DSB sampler are defined as:

$$
t_{m}=t_{m-1}+\alpha_{m}=t_{0}+\sum_{i=1}^{m} \alpha_{i}
$$

where $1 \leq m \leq \mathcal{N}, E\left[t_{m}\right]=m T$ and $\operatorname{var}\left[t_{m}\right]=m \sigma^{2} \forall \mathcal{N} \geq 1$. Note that $\left\{\alpha_{m}\right\}$ is a set of i.i.d random variables with probability density $p_{1}(\tau)$, mean $T$ and 125 with variance $\sigma^{2}$. Although, the input components of the DSB sampler have changed compared to the regular MC sampler but the signal at the output of the NUS remains the same as in the case of MC sampling [? ]. As a result, for each $L$ uniformly spaced samples in case of Nyquist sampling, we get $p$ non-uniform samples (see Fig 3 ).

Let $\mathcal{T}=\left\{\tau_{i}\right\}_{i=0}^{p-1}$ be the set of the durations between $p$ adjacent samples, where $\tau_{i-1}=c_{i}-c_{i-1}$ for $1 \leq i \leq p-1$ and $\tau_{p-1}=\left(L+c_{0}\right)-c_{p-1}$ (see Fig. 3). 


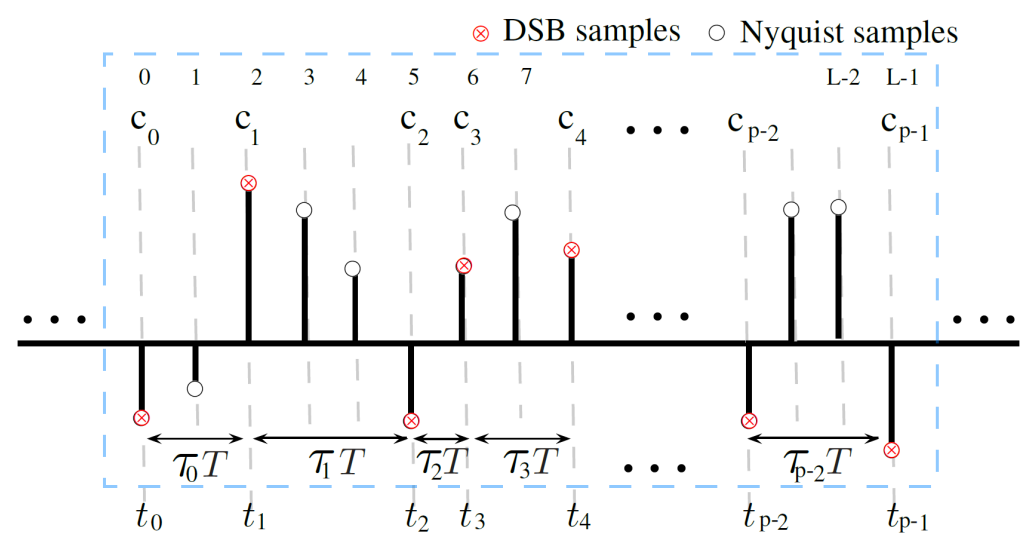

Figure 3: $L$ uniformly spaced Nyquist samples and corresponding $p$ DSB samples.

The set $\mathcal{C}=\left\{c_{i}\right\}_{i=0}^{p-1}$ contains $p$ distinct integers from $\{0,1, \ldots, L-1\}$. By taking $t_{0}=c_{0} T$, (3) can be written as

$$
t_{m}=c_{0} T+T \sum_{i=1}^{m} \tau_{i-1} \quad 1 \leq m \leq p .
$$

The set of sampling instants $\left\{t_{n}\right\}_{n \in \mathbb{Z}}$ is non-uniform and periodic with period $L$ like the regular MC sampler. But unlike the regular MC sampler, the DSB sampler uses a non uniform sampler witch samples only some portions of the received signal. It can be possible to switch off the ADC on a predefined instances to reduce the power consumption. Most of the existing non uniform digitizer use a uniform clock as a reference clock, and have some circuitry which transforms the uniform clock to a non uniform one [? ? ].

Following the approach of the MC sampling presented in Fig. 1. we express the non-uniform samples $x\left(t_{n}\right)$ at the output of the NUS in terms of periodic sequences $x_{i}[m]=x\left(t_{m p+i}\right)$, where $0 \leq i \leq p-1$. The Fourier transform, $X_{i}(f)$, of the sampled sequence $x_{i}[n]$ is related to the Fourier transform $X(f)$ of the unknown signal $x(t)$ by the following equation [? ] :

$$
X_{i}(f)=\frac{1}{L T} \exp \left(j 2 \pi f c_{i} T\right) \sum_{n=-\frac{L}{2}}^{\frac{L}{2}-1} X\left(f+\frac{n}{L T}\right) \exp \left(j 2 \pi \frac{c_{i}}{L} n\right)
$$

where $0 \leq i \leq p-1$, and $f \in \mathcal{B}_{0}=\left[0, \frac{1}{L T}\right]$. Note that $x(t) \in \mathcal{M}(\mathcal{B})$.

The expression in (5) can be written in a matrix form

$$
\mathbf{y}(f)=\mathbf{A}_{\mathcal{C}} \mathbf{s}(f)
$$

where $f \in \mathcal{B}_{0}$ and $\mathbf{y}(f)$ is a vector of size $p \times 1$ whose $i^{t h}$ element is given by

$$
y_{i}(f)=X_{i}(f) \exp \left(-j 2 \pi f c_{i} T\right) .
$$


$\mathbf{A}_{\mathcal{C}}$ is a matrix of size $p \times L$ whose $(i, l)^{t h}$ element is given by [? ]

$$
\left[\mathbf{A}_{\mathcal{C}}\right]_{i l}=\frac{1}{L T} \exp \left(j 2 \pi \frac{c_{i}}{L}\left(l-\frac{L}{2}\right)\right),
$$

where $0 \leq l \leq L-1$. Note that $\mathbf{s}(f)$ in (6) represents the unknown vector of size $L \times 1$ whose $l^{t h}$ element is given by

$$
s_{l}(f)=X\left(f+\frac{l-\frac{L}{2}}{L T}\right) .
$$

(9) indicates that, if the spectrum $X(f)$ is divided into $L$ cells indexed from $-\frac{L}{2}$ to $\frac{L}{2}-1$, then each cell corresponds to a line in the vector $\mathbf{s}(f)$, for $f \in \mathcal{B}_{0}$.

\subsection{Non-Uniform Spectrum Sensing Block (NUSSB)}

The reconstruction of $x(t)$ requires to solve (6). Using the fact that $x(t)$ is sparse in the frequency domain and that there is no energy in certain cells, the number of unknowns in (6) can be reduced. The set $\mathcal{K}$ consists of the spectral indexes of the cells that contain signal which are called the active cells. But in order to find $\mathcal{K}$ different methods have been proposed in literature [? ? ? ]. But the limitation of the methods proposed is that they require complete knowledge of the matrix $\mathbf{A}_{\mathcal{C}}$, the number of bands $N$ and the maximum bandwidth. Since we assume that we do not have any information regarding the spectrum of the signal $x(t)$, therefore, the matrix $\mathbf{A}_{\mathcal{C}}$, along with other parameters, cannot be known. In order to overcome this hurdle, we treat this scenario as a missing data problem. In [? ] we have proposed to use the Lomb-Scargle's method [? ] to estimate the power spectral density (PSD) of the non-uniformly sampled signal, in spectrum sensing. In this paper, rather than Lomb-Scargle periodogram, we propose to use the well know Non-Uniform Fourier Transform through Bartlett's method [?]. The PSD obtained for $N_{s}=K p M$ samples $x\left(t_{n}\right)$ taken at times $t_{n}, n=0, \ldots, N_{s}-1$, is defined by 100 :

$$
\hat{P}_{\text {Bart }}(\omega)=\frac{1}{N_{s}} \sum_{i=0}^{K-1}\left|\sum_{m=0}^{p M-1} x\left(t_{m}+i M L T\right) \exp \left(-j \omega t_{m}\right)\right|^{2}
$$

where $\omega=2 \pi f$ with $f \in\left[0, \frac{1}{2 T}\right], M>0, K>0$ and $0<p \leq L$.

Once the PSD is estimated, the threshold value, $\eta$, is selected dynamically as a function of $P S D_{\max }$ i.e. $\eta=\left\lfloor P S D_{\max }-\beta\right\rfloor$, where $\beta$ is a fixed value and $\lfloor *\rfloor$ is the floor function. With reference to $\eta$, the number of bands $N$ and the spectral 145 support $\mathcal{F}$ are computed. The block diagram of our non-uniform spectrum sensing model is presented in (Fig. 4). Once the support $\mathcal{F}$ and $N$ are found, the set of spectral indexes $\mathcal{K}=\left\{k_{r}\right\}_{r=0}^{q}$, where $k_{r} \in\left\{-\frac{L}{2},-\frac{L}{2}-1, \ldots, \frac{L}{2}-1\right\}$, can be calculated using (11) as follows

$$
\left\lfloor a_{i} L T\right\rfloor \leq \kappa_{i} \leq\left\lfloor b_{i} L T\right\rfloor
$$




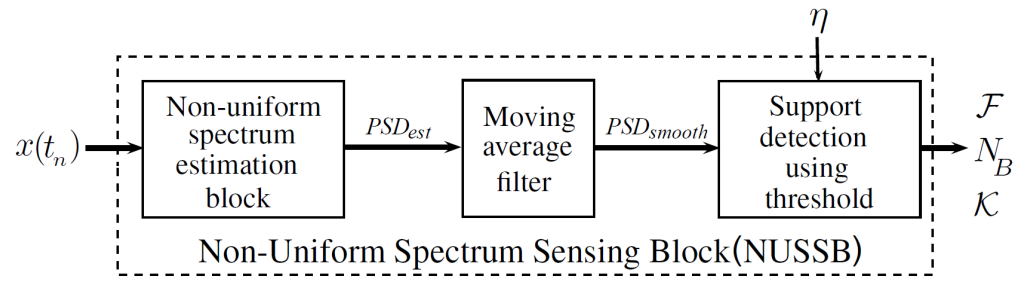

Figure 4: Non-uniform spectrum sensing model

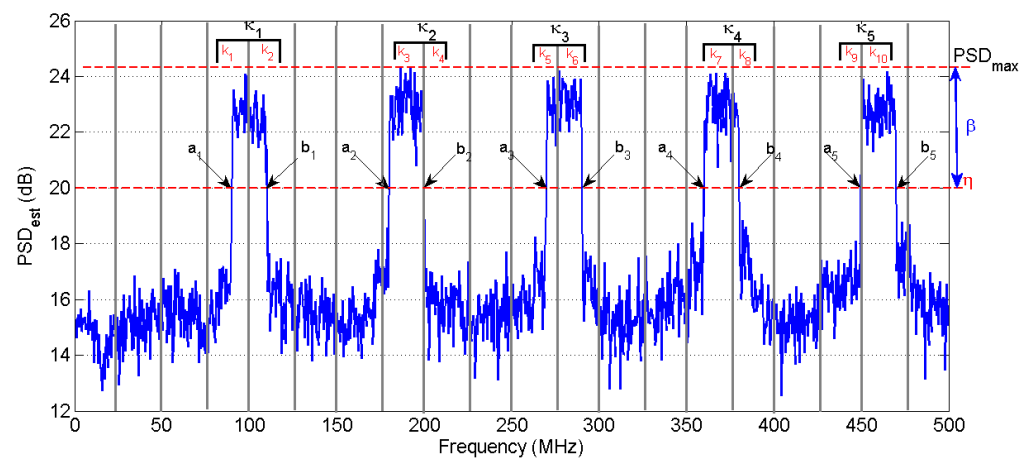

Figure 5: Support detection using threshold in non-uniform spectrum sensing block.

where $1 \leq i \leq N$ and $\lfloor *\rfloor$ is the floor function. Once all the $\kappa_{i}$ are calculated for each band, the set of spectral indexes is given by :

$$
\mathcal{K}=\bigcup_{i=1}^{N} \kappa_{i}
$$

The process is illustrated in Fig 5 for a signal with $N=5$.

150 multi-coset reconstruction blocks, as shown in (Fig 2).

The performance of our Non-Uniform Spectrum Sensing Block is evaluated by computing the probabilities of the signal occupancy in terms of the number of bands $N$ and the active cells set $\mathcal{K}$ as follows:

1. The good detecting probability

$$
\begin{array}{r}
P_{d}(N)=\operatorname{Pr}(\hat{N}=N) \\
P_{d}(\mathcal{K})=\operatorname{Pr}(\hat{\mathcal{K}}=\mathcal{K})
\end{array}
$$

2. and the false alarm probability

$$
\begin{array}{r}
P_{f a}(N)=\operatorname{Pr}(\hat{N}>N) \\
P_{f a}(\mathcal{K})=\operatorname{Pr}(|\hat{\mathcal{K}}|>|\mathcal{K}| \mid \mathcal{K} \subset \hat{\mathcal{K}})
\end{array}
$$

155 where $|\mathcal{K}|$ represents the cardinality of $\mathcal{K}$. $\hat{\mathcal{K}}$ and $\hat{\mathcal{N}}$ represent, respectively, the estimate of $\mathcal{K}$ and $\mathcal{N}$ given by the NUSSB. 
In our proposed NUSSB, the threshold, $\eta$, is the only information assumed to be available on the input signal. It should be noted that these technique can be used only if the power difference between each band is small.

As will be shown later in the paper through simulations, the results obtained using Bartlett's method for spectral support detection are quite accurate.

\subsection{Multi-Coset Reconstruction Block}

After having found the set $\mathcal{K}$, the vector $\mathbf{s}(f)$ is reduced in dimension to $\mathbf{z}(f)$, a vector of size $q \times 1$ with $r^{t h}$ element given by $(17)$ :

$$
z_{r}(f)=X\left(f+\frac{k_{r}}{L T}\right) .
$$

The matrix $\mathbf{A}_{\mathcal{C}}$ becomes $\mathbf{A}_{\mathcal{C}}(\mathcal{K})$, a matrix of size $p \times q$

$$
\left[\mathbf{A}_{\mathcal{C}}(\mathcal{K})\right]_{i r}=\frac{1}{L T} \exp \left(j 2 \pi \frac{c_{i}}{L} k_{r}\right)
$$

where $0 \leq i \leq p-1$ and $0 \leq r \leq q-1$. As a result (6) is reduced to

$$
\mathbf{y}(f)=\mathbf{A}_{\mathcal{C}}(\mathcal{K}) \mathbf{z}(f)
$$

As our non uniform sampler (see 3.1 is reconfigurable, we always take $p=q$. So, the matrix $\mathbf{A}_{\mathcal{C}}(\mathcal{K})$ is a full column rank matrix, the unique solution of $(6)$ is given by 20 .

$$
\mathbf{z}(f)=\mathbf{A}_{\mathcal{C}}^{-\mathbf{1}} \mathbf{y}(f),
$$

where $\mathbf{A}_{\mathcal{C}}^{-1}$ is the inverse of $\mathbf{A}_{\mathcal{C}}(\mathcal{K})$ and $f \in\left[0, \frac{1}{L T}\right]$. Since $x(t) \in \mathcal{M}(\mathcal{B})$, its spectrum is Hermitian, the vector $\mathbf{z}(f)$ dimension can be reduce to $\mathbf{z}^{+}(f)$. Then (20) become (21)

$$
\mathbf{z}^{+}(f)=\mathbf{A}_{\mathcal{C}}^{+} \mathbf{y}(f)
$$

where $\mathbf{z}^{+}(f)$ is a vector of size $\frac{q}{2} \times 1$ with $r^{\text {th }}$ element given by 22 .

$$
z_{r}^{+}(f)=X\left(f+\frac{k_{r}}{L T}\right)
$$

where $1 \leq r \leq \frac{q}{2}$ and $\mathbf{A}_{\mathcal{C}}^{+}$, a matrix of size $\frac{q}{2} \times q$ whose $(i, l)^{t h}$ element is given by

$$
\left[\mathbf{A}_{\mathcal{C}}^{+}\right]_{i l}=2\left[\mathbf{A}_{\mathcal{C}}^{-1}\right]_{i l}
$$

where $0 \leq i \leq \frac{q}{2}-1$ and $0 \leq l \leq q-1$.

Once $\mathbf{z}^{+}(f)$ is found, the inverse Fourier transform achieves the time domain representation of each cell, $X\left(f+\frac{k_{r}}{L T}\right)$ Fourier transform of $x_{r}(t)=x_{r}^{\mathcal{R}}(t)+$ $j x_{r}^{\mathcal{I}}(t)$. The reconstructed signal in time domain is written as :

$$
\hat{x}(t)=2 \sum_{r=0}^{\frac{q}{2}-1} x_{r}^{\mathcal{R}} \cos \left(2 \pi \frac{k_{r}}{L T} t\right)-x_{r}^{\mathcal{I}} \sin \left(2 \pi \frac{k_{r}}{L T} t\right)
$$

The Multi-Coset reconstruction process is illustrated in (Fig,6) for $p M$ non uniform samples $x\left(t_{n}\right)$, taken at times $\left\{t_{n}\right\}_{n=0}^{p M-1}$, for $L M$, corresponding, Nyquist samples (see 2.2), with $M>0$. The total number of real operations after each stage is given in the 5 (TABLE1). 


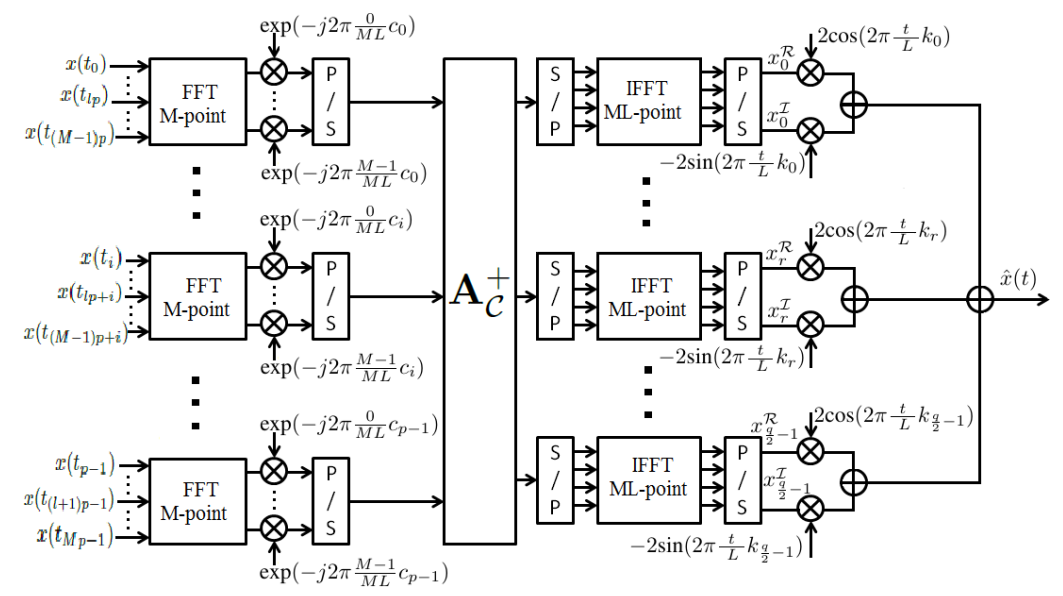

Figure 6: Multi-Coset Reconstruction block

\subsection{Complexity and power efficiency}

From (TABLE 1), the total number of real operations (addition and multiplication) to get a uniform Nyquist sample is given by (25) :

$$
\mathcal{N}_{\text {total }}=\frac{11 p}{2 L} \log _{2}(M)+\frac{37 p}{16} \log _{2}(M L)+\frac{9 p^{2}}{2 L}+\frac{6 p}{L}+\frac{p^{2}}{4}+\frac{p}{2}
$$

Since we have defined our NUS (see. 3.1) as an ADC which can be switch off at predefined instances and have some circuitry which transforms the uniform clock to a non uniform one. Assuming insignificant the power consumption to transforms the uniform clock. We define $E_{d s b}$ as the energy consumed by our DSB system to get a Nyquist sample by (26)

$$
E_{d s b}=\tau E_{u s}+\mathcal{N}_{\text {total }} P_{c p u}
$$

where $\tau=(p / L)$ is the compression ratio, $E_{u s}$ represents the energy consumed by the ADC for a Nyquist sample and $P_{c p u}$ is the power efficiency of the chosen processor, measured in Joule per Operations $(\mathrm{J} / \mathrm{OP})$. The proposed system is efficient only if $E_{d s b}<E_{u s}$, then the processor power efficiency must be chosen as (27)

$$
P_{\text {cpu }}<(1-\tau) \frac{E_{u s}}{\mathcal{N}_{\text {total }}}
$$

For example, if $p=10, L=128, M=512$ then $\tau \approx 0.078$, we select a 14-bits 100 MSPS digitally calibrated pipeline ADC which consumes $250 \mathrm{~mW}$ per 100 MSPS [? ] then, $E_{u s}=2,5 \mathrm{~nJ}$ and $\mathcal{N}_{\text {total }} \approx 408$ operations. So from (27), we deduce $P_{c p u}<2.305 \mathrm{~nJ} / \mathrm{OP}$. Furthermore, to maintain a real-time processing, the execution time of the $\mathcal{N}_{\text {total }}$ operations must be less than the sampling period. Then the chosen processor in this example must be performs more than 40.8 Giga operations per second. It is shown in [? ] a processor which 


\begin{tabular}{|c|c|c|}
\hline & Multiplication & Addition \\
\hline $\begin{array}{c}\text { Radix-2 Butterfly FFT M-point } \\
(\mathrm{M}, \text { taken as a power of } 2)\end{array}$ & $2 p M \log _{2}(M)$ & $\frac{7}{2} p M \log _{2}(M)$ \\
\hline $\begin{array}{c}\text { Multiplication by } \exp \left(-j 2 \pi \frac{l}{M L} c_{i}\right) \\
\text { when } l=\{0,1, \ldots, \mathrm{M}-1\}, i=\{0,1, \ldots, \mathrm{p}-1\}\end{array}$ & $4 p M$ & $3 p M$ \\
\hline Multiplication by Matrix $\mathbf{A}_{\mathcal{C}}^{+}$ & $2 p^{2} M$ & $\frac{5}{2} p^{2} M-p M$ \\
\hline $\begin{array}{c}\text { Radix-4 Butterfly IFFT ML-point } \\
\text { (ML, taken as a power of } 4)\end{array}$ & $\frac{3}{4} p M L \log _{2}(M L)$ & $\frac{25}{16} p M L \log _{2}(M L)$ \\
\hline $\begin{array}{c}\text { Multiplication by } \\
2 \cos \left(2 \pi \frac{k_{r}}{L} t\right) \text { when } t=\{0,1, \ldots, \text { ML-1 }\}, \\
k_{r}=\left\{k_{0}, k_{1}, \ldots, k_{\frac{q}{2}}\right\}\end{array}$ & $\frac{1}{2} \mathrm{pML}$ & 0 \\
\hline $\begin{array}{c}\text { Multiplication by } \\
-2 \sin \left(2 \pi \frac{k_{r}}{L} t\right) \text { when } t=\{0,1, \ldots, \text { ML-1 }\}, \\
k_{r}=\left\{k_{0}, k_{1}, \ldots, k_{\frac{q}{2}}-1\right\}\end{array}$ & $\frac{1}{2} \mathrm{pML}$ & 0 \\
\hline Sum & & $\frac{1}{4} p^{2} \mathrm{ML}-\frac{1}{2} p \mathrm{ML}$ \\
\hline
\end{tabular}

Table 1: Number of real operation in Multi-Coset Reconstruction block

processes at 50 Giga operations per second with a power efficiency around 57,8 fJ/OP. Then our system could be more power efficiency than regular ADC when the bands locations are unknown with a very sparse signal.

\subsection{Optimal Average Sampling Rate Search Block (OASRSB)}

The ideal sampling pattern is obtained when the condition number of $\mathbf{A}_{\mathcal{C}}(\mathcal{K})$ is as small as possible, [? ? ]. The condition number of a matrix $U$ is defined as $\operatorname{cond}(U)=\|U\|\left\|U^{-1}\right\|$ where $\|*\|$ is a norm operation. The choice of $\mathcal{C}$ can be seen as the solution to the following minimization problem

$$
\mathcal{C}_{\text {opt }}=\arg \min _{\mathcal{C}:|\mathcal{C}|=p} \operatorname{cond}\left(\mathbf{A}_{\mathcal{C}}(\mathcal{K})\right)
$$

One way of solving (28) is to use greedy search algorithms. Authors in [? ] proposed to use the Sequential Forward Selection (SFS) to find the set $\mathcal{C}$. The SFS algorithm used in [? ] lacks the capability to remove elements from set $\mathcal{C}$ that become obsolete after the addition of other elements. For a given set $\mathbb{L}=0,1, \ldots, L-1$, the SFS looks for a subset $\mathcal{C}=\left\{c_{i}\right\}_{i=1}^{p}$ which minimizes the function $\operatorname{cond}\left(\mathbf{A}_{\mathcal{C}}(\mathcal{K})\right)$. $L, T$ and $\mathcal{K}$ known, SFS algorithm searches for an optimal sampling pattern $\mathcal{C}$ which in turn minimizes the reconstruction error. Finally, $\mathcal{C}$ is used to compute the elements of the set $\mathcal{T}$. Thus, for a given $L$, the non-uniform sampler operates at an optimal average rate which depends only on the number of active bands. As a result, the average sampling rate $\bar{f}$ of our proposed sampler is given by :

$$
\bar{f}=\frac{p}{L T}=\frac{|\mathcal{K}|}{L T}
$$




\section{Numerical Results}

For simulations, we consider a multi band signal with a variable number of bands $N \in\{1,2, \ldots, 6\}$, each with a bandwidth $\mathrm{B}=20 \mathrm{MHz}$, we assume that the $N$ bands have the same power. The wide band of interest is in the range of $[0,500] \mathrm{MHz}$ i.e. $f_{n y q}=1 \mathrm{GHz}$. $16 \mathrm{QAM}$ modulation symbols are used that are corrupted by the additive white Gaussian noise. To evaluate the performance of our Dynamic Non-uniform Sampler, we start by presenting in Sub-Section 4.1. the performance of our Non-Uniform Spectrum Sensing block versus to $\alpha=p / L$ ( the compression ratio), $\beta$ (the threshold) and the SNR. In the Sub-Section 4.2 . we illustrate the performance of the proposed DSB sampler by comparing it to 190 regular MC scheme and the drawback of poor detection of the spectral support on its average frequency.

\subsection{Performance of Bartlett Method}

In this sub-section we will evaluate the performance of our NUSSB in terms of good detection of the number of bands $N$ and the set of actives bands $\mathcal{K}$ (see.(3.2). For simulation, we take $L=128, M=16$ and $K=32$ as parameters in equation (10), and use SFS algorithm to choose the sampling instances.

In order to compute $P_{d}$ and $P_{f a}$, we start by choosing an appropriate $\beta$. In Fig.7, we have performed 1000 iterations at $\mathrm{SNR}=10 \mathrm{~dB}$ and for different values of $\alpha$ and $\beta$. It can be seen here that whatever $\beta, P_{d}(\mathcal{K})$ increases with increasing $\alpha$ until reach a maximum at some $\beta$ values. These values are reached around $\beta=-6,-5.5$ and $-5 \mathrm{~dB}$ respectively for $\alpha=0.7,0.6$ and 0.5 . In the rest of the simulation, we take $\beta=-6,-5.5$ and $-5 \mathrm{~dB}$ respectively for $\alpha=0.7,0.6$ and 0.5. In Fig 8 , we plot the $P_{f a(N)}$ and $P_{f a(\mathcal{K})}$ as a function of varying SNR.

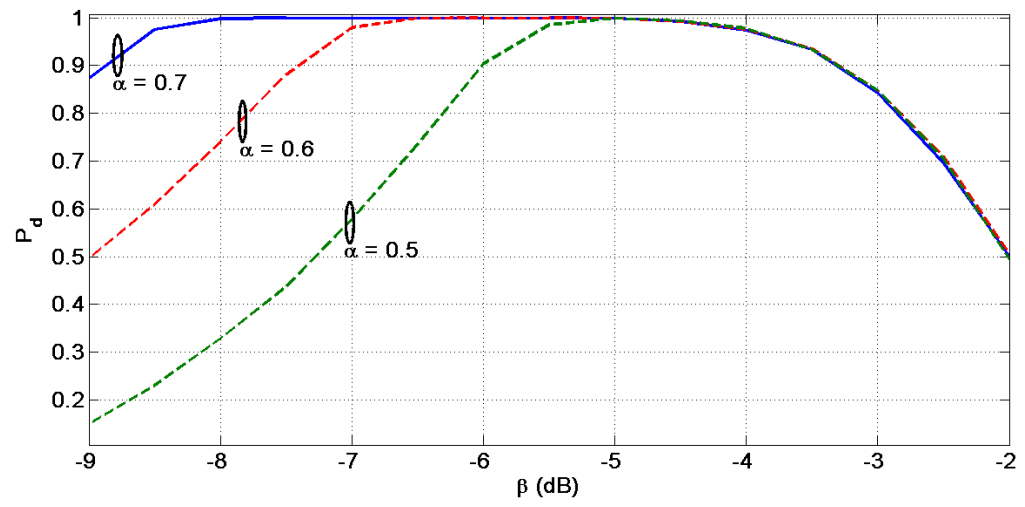

Figure 7: $P_{d}(\mathcal{K})$ plotted against varying $\beta$ at $\mathrm{SNR}=10 \mathrm{~dB}$

At low SNR i.e. $-2 \mathrm{~dB}$, the values for $P_{f a(N)}$ and $P_{f a(\mathcal{K})}$ are quite high, especially for $\alpha \geq 0.5$. But as the SNR increases, the $P_{f a(N)}$ and $P_{f a(\mathcal{K})}$ drop quickly, practically becoming zero at $\mathrm{SNR}=6 \mathrm{~dB}$ for $\alpha=0.7$. As expected, $P_{f a(N)}$ and $P_{f a(\mathcal{K})}$ also depend on the number of non-uniform samples available to NUSSB for detection. 


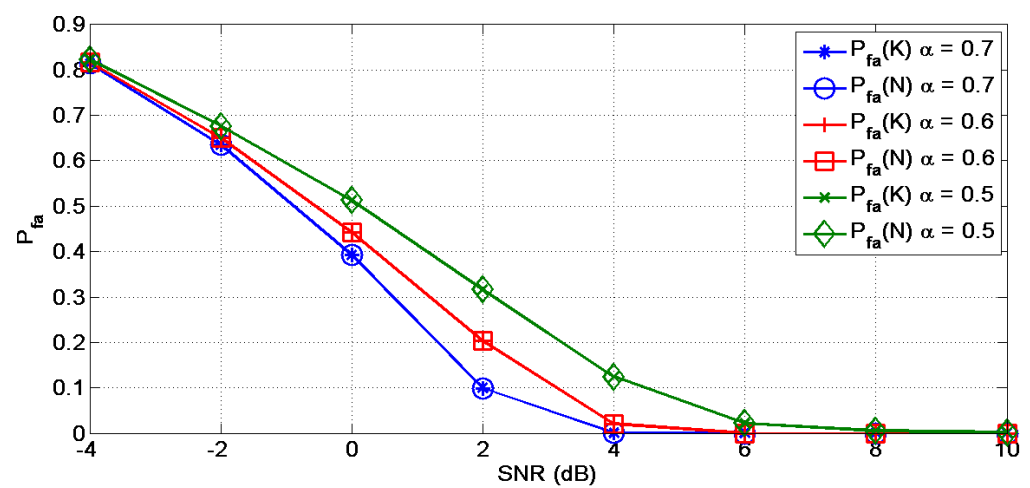

Figure 8: $P_{f a(N)}$ and $P_{f a(\mathcal{K})}$ plotted against varying SNR for $\alpha=0.5$ to $\alpha=0.7$.

As the average sampling rate for the DSB sampler is $f_{\text {avg }}=(p / L) f_{n y q}$, it can be suspected that a false estimation of $N, \mathcal{K}$ by NUSSB will directly effect the $f_{\text {avg }}$ of the DSB sampler. This can happen because at a high $P_{f a}$, the NUSSB shows that the estimated spectral support $\mathcal{F}$ of the signal is larger than the actual support, as a result, the DSB sampler is forced to sample at a higher, unoptimized sampling rate. This will be illustrated in the next sub-section.

\subsection{Flexibility and adaptivity of $D S B$}

To start, we assume that the traditional MC sampler is designed not only to have an optimal reconstruction for the signals but also a perfect knowledge of $(N, \mathcal{K})$ of the incoming signal while on the other hand, our proposed DSB sampler operates in blind mode and therefore has no information regarding the $\mathcal{F}$ and $N$ of any kind of signals. In the following, we use the subscripts $m c$ and $d s b$ to differentiate between simulation parameters for MC and DSB samplers, respectively. It should be noted that $p_{m c}$ allows to set the number of branches [? ] or number of uniform sampler placed in parallel [? ? ] on the MC sampler. For sampling process, we select $L=128$ and let the samplers select their respective parameter $p_{m c} / p_{d s b}$ depending on the signal's sparsity. We design the MC sampler so as to have an optimal reconstruction for wide band signals $x(t)$ with frequencies carriers $f_{i} \in\{15,170,250,330,410,490\}$, each band of bandwidth $B=20 \mathrm{MHz}$, the set of spectral indexes representing the set of actives cells $\mathcal{K}$ is given by (11) and $(12)$, and the sampling pattern $\mathcal{C}$ is computed using SFS algorithm [? ]. Finally we have $p_{m c}=50$. We measure the performance of MC sampler and DSB sampler in terms of the Root Mean Square Error (RMSE) of the reconstructed time domain signal $\hat{x}(t)$. It is defined by $(30)$ :

$$
\mathrm{RMSE}=\frac{\|\hat{x}(t)-x(t)\|_{2}}{\|x(t)\|_{2}}
$$

In Fig 9, we have plotted the RMSE as a function of condition number of $\mathbf{A}_{\mathcal{C}}(\mathcal{K})$ ) of signal $x(t)$ without noise. The sampling pattern $\mathcal{C}$ is chosen randomly and by using the SFS algorithm (see. 3.5). It can be seen that SFS Algorithm make the reconstruction more efficient than a randomly choice of the set $\mathcal{C}$. 


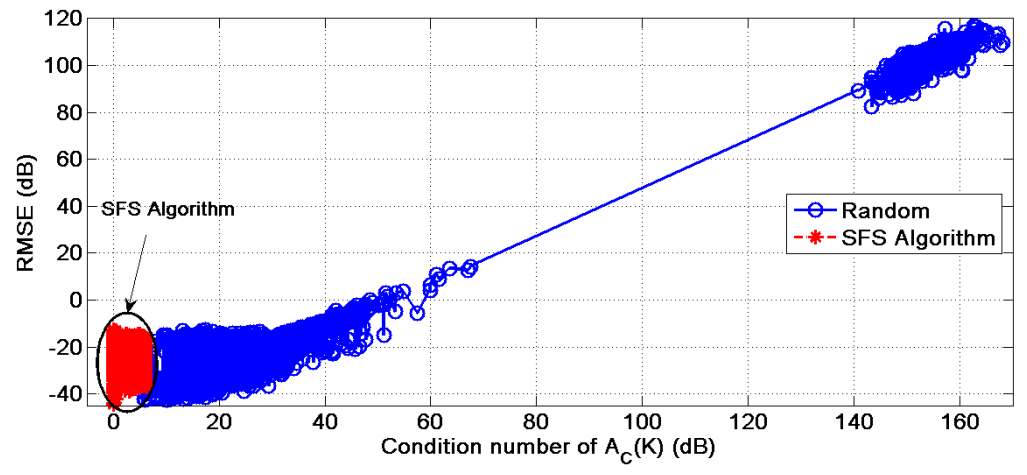

Figure 9: RMSE as function of Condition number of $A_{\mathcal{C}}(\mathcal{K})$ (no noise) sampler remain unchanged due to the hardware rigidity regardless of the input signal characteristics. Since the spectral support is unknown to the DSB sampler, it always starts from the adaptation phase to find $\mathcal{F}$ and $N$, and then goes 225 ahead to operate in reconstruction phase until needed to recheck the spectrum of the input signal. The sampler can be programmed to periodically check the spectral changes. For simplicity we assume here that the instants of changes in spectrum are known. So, the sampler swhiches to the adaptation phase each time there is a change in the spectrum of the input signal to detect the new support.

Fig, 10, shows that the performance of our proposed blind DSB sampler completely matches with the non-blind MC sampler in terms of RMSE. The

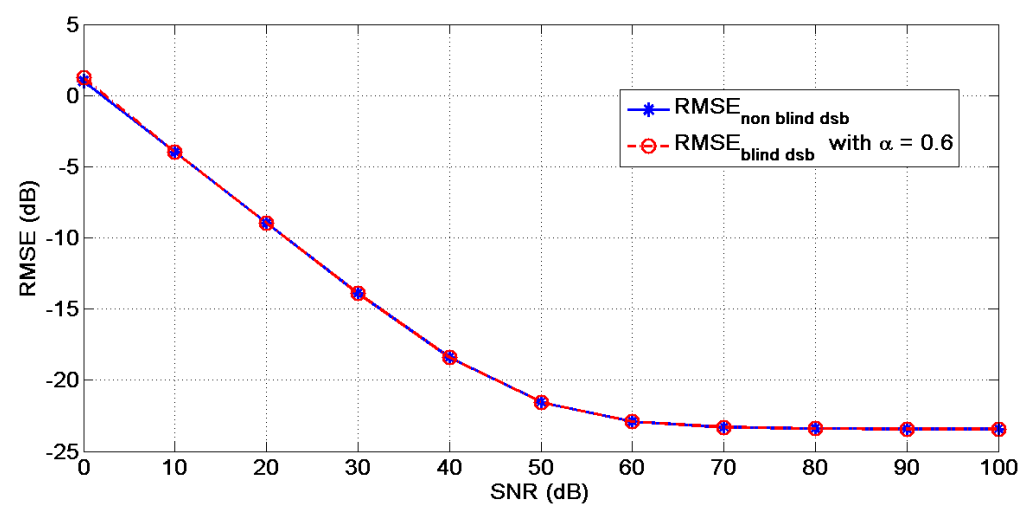

Figure 10: Comparison between our proposed blind DSB sampler with non-blind DSB sampler in terms of RMSE.

results obtained are very promising. We can conclude from Fig 10 that the Bartlett method used for support detection makes our proposed sampler same 
235 as the non-blind one as. However, it is noted that such convincing results are obtained with a very sparse multiband signal.

In order to show the flexibility and adaptivity of the proposed DSB sampler, we consider that the spectrum of the signal $x(t)$ changes with time. In other word the number of bands $N$ varies from 1 to 7 . All the results provided in 240 Figs $11,12,13$, are for the scenario where the number of bands $N$ changes from $6 \rightarrow 4 \rightarrow 2 \rightarrow 1 \rightarrow 3 \rightarrow 5 \rightarrow 7 \rightarrow 6$. For simplicity we assume that the change in $N$ occurs after every 2 time instants i.e. at time instants 1 and $2, N=6$, for time instants 3 and $4, N=4$ and $N$ and so on. The evolution of $N$ with time is indicated at the top of each of the Figs 11$] 12,13$.

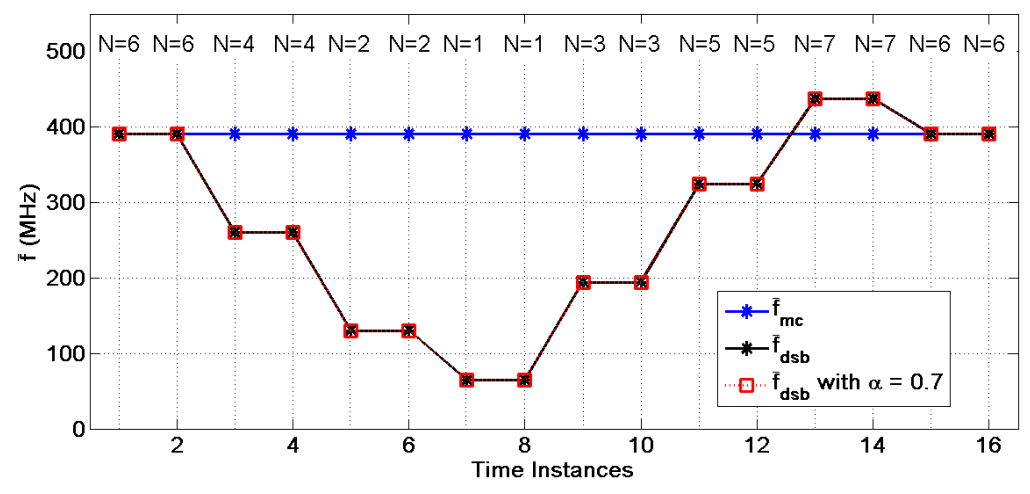

Figure 11: Variation in $\bar{f}_{m c}$ and $\bar{f}_{d s b}$ as $N$ changes over time at an SNR $=50 \mathrm{~dB}$.

At the start when the time instant is equal to 1 , the samplers start with $N=6$. Based on the signal spectrum characteristics, both MC and DSB samplers start sampling at $\bar{f}_{m c}=\bar{f}_{d s b}=\bar{f}_{d s b}$ with $\alpha=0.7 \simeq 390.62 \mathrm{MHz}$ with $\bar{p}_{m c}=\bar{p}_{d s b}=\bar{p}_{d s b \text { with } \alpha=0.7}=50$, (see Figs $11-12$. Note that $\bar{f}_{m c}, \bar{f}_{d s b}$ and $\bar{f}_{d s b \text { with } \alpha=0.7}$ represent the average sampling frequencies for non blind MC,

250 non blind DSB samplers and blind DSB with $\alpha=0.7$ respectively. When $N$ changes from $6 \rightarrow 4$ at time instant 3 , our DSB sampler adapts accordingly by recalculating the signal support using Bartlett method in the adaptation phase. The updated signal support is then fed to the non-uniform sampler block. As shown in Fig 11, at $N=4$ the new updated average sampling frequency for our DSB sampler reduces to $\bar{f}_{d s b} \simeq 260.31 \mathrm{MHz}$ with $\bar{p}_{d s b} \simeq 34$. Since the MC sampler lacks from an adaptive feedback mechanism, it continues to sample at $f_{m c} \simeq 390.62 \mathrm{MHz}$ with $p_{m c}=50$ depicted in Fig. 12. Fig.11, shows that as $N$ decreases to 2 , the $\bar{f}_{d s b}$ is further reduced to $130.47 \mathrm{MHz}$ which is $13 \%$ of $f_{n y q}=1 \mathrm{GHz}$ with $\bar{p}_{d s b} \simeq 9$ shown in Fig. 12. On the other hand, MC sam260 pler continues to operate at $\bar{f}_{m c} \simeq 390.62 \mathrm{MHz}$ with $p_{m c}=50$ even for $N=2$ what, clearly depicts a sub-optimal performance compared to the proposed DSB sampler. This shows that our system adapts itself dynamically and reduces $\bar{f}_{d s b}$ which results in reduced number of incoming samples $p_{d s b}$ that are to be treated later. At the opposite, when $N$ increases from $2 \rightarrow 3$, the proposed DSB sampler adapts accordingly by re-evaluating the new support through the Bartlett 


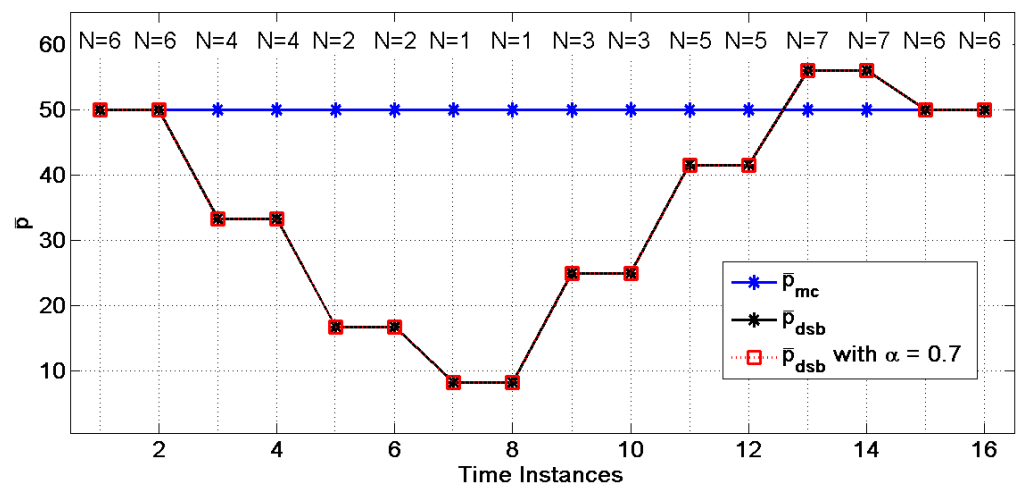

Figure 12: Variation in $p_{m c}$ and $p_{d s b}$ as $N$ changes over time at an SNR $=50 \mathrm{~dB}$

method, as explained earlier, and adjusts $\bar{p}_{d s b}$ and $\bar{f}_{d s b}$, as shown in Figs 11 12

In Fig 13, we have plotted the variation in RMSE, for the two samplers, corresponding to Figs.11 12, as $N$ changes over time. If we closely analyze Fig 13 , we observe that $R M S E_{m c}$ is slightly better compared to $R M S E_{d s b}$ when $N$ decreases from $6 \rightarrow 4 \rightarrow 2$.

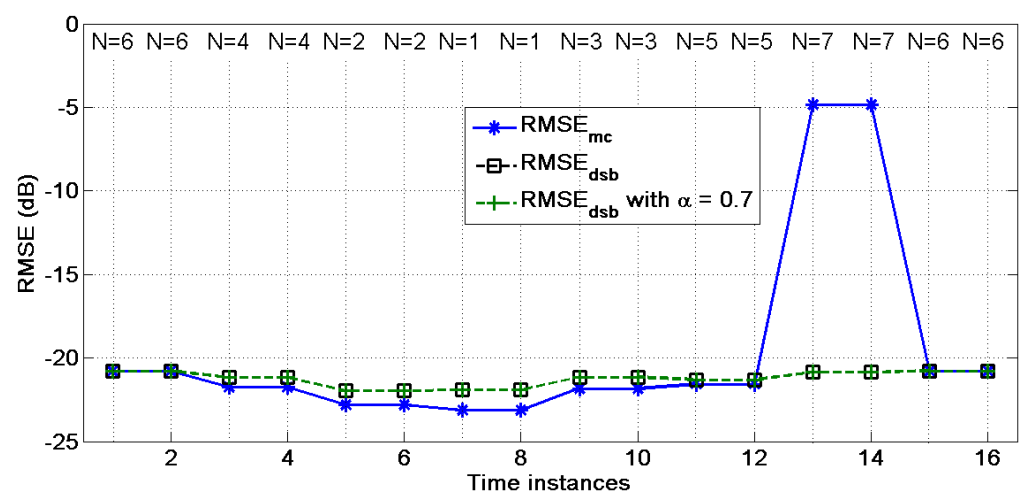

Figure 13: Variation in $R M S E_{m c}$ and $R M S E_{d s b}$ as $N$ changes over time at an $\mathrm{SNR}=50 \mathrm{~dB}$

270

This happens because the MC sampler uses much more samples than the proposed one. The most important variation in $R M S E_{m c}$ to observe is when $N$ changes from $5 \rightarrow 7$. Fig. 12 shows that as $N$ changes from $5 \rightarrow 7$, our DSB sampler adjusts $p_{d s b}$ to 56 . While for the MC sampler, $p_{m c}$ remains equal to the input of the MC sampler. This results in a drastic increase in $R M S E_{m c}$ i.e. of the order of $-5 \mathrm{~dB}$ as shown in Fig 13 . Even if we suppose that there is a feedback mechanism in the $\mathrm{MC}$ sampler to set $p_{m c}=56$, it cannot adjust $p_{m c}$ because of the hardware limitations, i.e. the MC sampler used in our simulation has only $p=50$ branches. On the other hand, adjust $p_{d s b}=56$ and the $R M S E_{d s b}$ remains low i.e. of the order of $-21 \mathrm{~dB}$. Therefore, our DSB sampler adapts 
quickly to avoid any loss of data. The proposed Adaptive non uniform sampler uses a single ADC which can be switched off at predefined instances and can theoretically adapt $p_{d s b}$ to any value up to 128 depending on the signal.

In Fig 14 $F_{\text {avg-opt }}$, represents the optimal average sampling rate of DSB sampler when operating in non blind mode i.e. having full information of the spectral support. When the DSB sampler operates in blind mode, $F_{\text {avg }}=F_{n y q}$ represents the worst case and the ideal case is when $F_{\text {avg }}=F_{\text {avg-opt }}$.

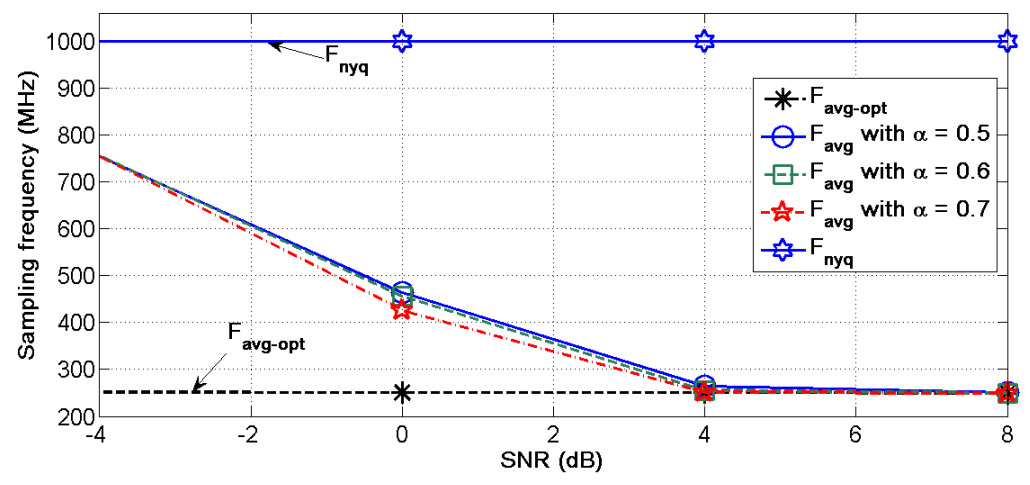

Figure 14: Average sampling estimation rate $F_{\text {avg }}$ of DSB sampler while operating in blind mode plotted for different $\alpha$

It should be noted that the results in Figs.14,15, show the impact of false detection $\left(P_{f a}(\mathcal{K})\right)$, performed in the adaptation phase, on the average sampling rate achieved in the reconstruction phase. In Fig 14 it can be shown than the SNR impacts the averaging sampling rate $F_{a v g}$ of the DSB sampler. This happens because at low SNR the false alarm probability is high. Then the NUSSB shows the estimated spectral's estimated support $\mathcal{K}$ as larger than the actual support, this make the DSB sampler to sample at a higher, un-optimized sampling rate. This becomes more clear Fig,15 where $F_{\text {avg }}$ is plotted as a function of $P_{f a}(\mathcal{K})$ for different $\alpha$. It can be seen that with the increase of $P_{f a}(\mathcal{K}), F_{\text {avg }}$ increases, whatever $\alpha$, and approaches $F_{n y q}$ at $P_{f a}(\mathcal{K})=1$.

\section{Conclusion}

In this paper we proposed a new smart sampling system for cognitive radio, called the Dynamic Single Branch non-uniform sampler. To ensure optimal reconstruction with a small number of samples, the DSB adapts its parameters according to the input signal's sparsity. Its operates in two phases, the adaptation phase and the reconstruction phase. In the adaptation phase, the

305 proposed scheme senses the spectrum and adapts its sampling rate by computing the proposed non-uniform spectrum sensing technique. We have shown that the proposed sensing model works efficiently and shows high detection and low false alarm probabilities. The performance of the spectrum sensing model improves by increasing the number of available non-uniform samples to the sensing 


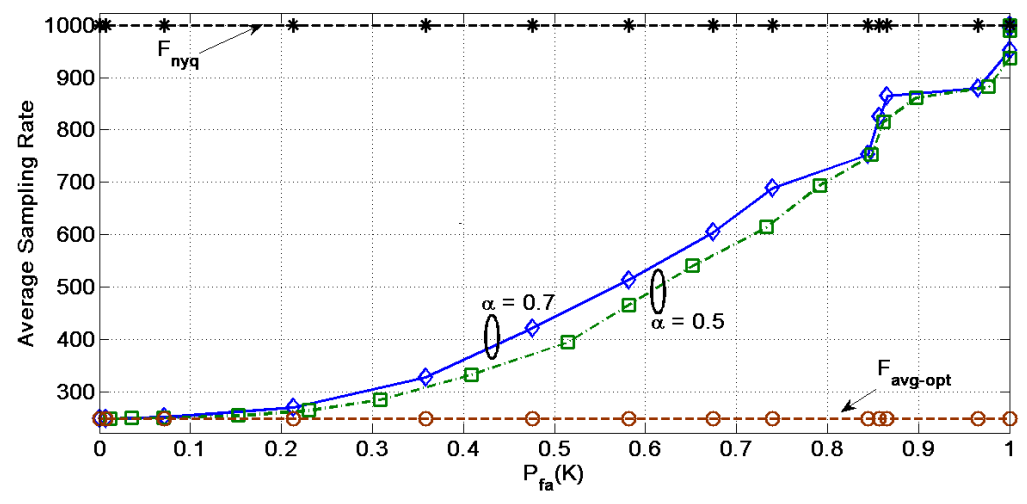

Figure 15: Estimation average sampling rate $F_{a v g}$ plotted against $P_{f a}(\mathcal{K})$ for different $\alpha$

310

method. As the sampling instances of our Non-uniform sampler depends to the position of this one on the Nyquist grid, then the average sampling rate depends on the number of bands contained in the signal. Furthermore, we have shown the effect of false detection on it. The DSB sampler performance has been compared to those of a traditional, non flexible Multi-Coset architecture which contains severals parallel uniform samplers. We have shown that our system is significantly more efficient than the conventional MC sampler when the spectrum of signal changes. The system can be designed with existing ADC and falls in the category of software defined radio system.

\section{References}

[1] M. Mishali, Y. C. Eldar, From theory to practice: Sub-nyquist sampling of sparse wideband analog signals, Selected Topics in Signal Processing, IEEE Journal of 4 (2) (2010) 375-391.

[2] M. Rashidi Avendi, Non-uniform sampling and reconstruction of multi-band signals and its application in wideband spectrum sensing of cognitive radio.

[3] Y.-P. Lin, P. Vaidyanathan, Periodically nonuniform sampling of bandpass signals, Circuits and Systems II: Analog and Digital Signal Processing, IEEE Transactions on 45 (3) (1998) 340-351.

[4] R. Venkataramani, Y. Bresler, Optimal sub-nyquist nonuniform sampling and reconstruction for multiband signals, Signal Processing, IEEE Transactions on 49 (10) (2001) 2301-2313.

[5] Y. Bresler, Spectrum-blind sampling and compressive sensing for continuousindex signals, in: Information Theory and Applications Workshop, 2008, IEEE, 2008, pp. 547-554.

[6] M. Dominguez-Jitnenez, N. Gonzalez-Prelcic, Analysis and design of multirate synchronous sampling schemes for sparse multiband signals, in: Signal Processing 
Conference (EUSIPCO), 2012 Proceedings of the 20th European, IEEE, 2012, pp. 1184-1188.

[7] T. Moon, N. Tzou, X. Wang, H. Choi, A. Chatterjee, Low-cost high-speed pseudorandom bit sequence characterization using nonuniform periodic sampling in the presence of noise, in: VLSI Test Symposium (VTS), 2012 IEEE 30th, IEEE, 2012, pp. 146-151.

[8] S. Traore, B. Aziz, D. Le Guennec, Dynamic single branch non-uniform sampler, in: Digital Signal Processing (DSP), 2013 18th International Conference on, IEEE, 2013, pp. 1-6.

345 [9] S. Traore, B. Aziz, D. Le Guennec, Système d'échantillonnage non uniforme en radio intelligente, in: GRETSI 2013, 2013, p. nc.

[10] J. Palicot, Radio engineering: From software radio to cognitive radio, John Wiley \& Sons, 2013.

[11] M. Ben Romdhane, Echantillonnage non uniforme appliqué à la numérisation des signaux radio multistandard, Ph.D. thesis, Paris, ENST (2009).

[12] J. Liang, D. A. Johns, A frequency-scalable 15-bit incremental adc for low power sensor applications, in: Circuits and Systems (ISCAS), Proceedings of 2010 IEEE International Symposium on, IEEE, 2010, pp. 2418-2421.

[13] M. Rashidi, K. Haghighi, A. Owrang, M. Viberg, A wideband spectrum sensing method for cognitive radio using sub-nyquist sampling, in: Digital Signal Processing Workshop and IEEE Signal Processing Education Workshop (DSP/SPE), 2011 IEEE, IEEE, 2011, pp. 30-35.

[14] M. Rashidi, K. Haghighi, A. Panahi, M. Viberg, A nlls based sub-nyquist rate spectrum sensing for wideband cognitive radio, in: New Frontiers in Dynamic Spectrum Access Networks (DySPAN), 2011 IEEE Symposium on, IEEE, 2011, pp. 545-551.

[15] B. Aziz, S. Traore, D. Le Guennec, Non-uniform spectrum sensing for cognitive radio using sub-nyquist sampling, in: Signal Processing Conference (EUSIPCO), 2013 Proceedings of the 21st European, IEEE, 2013, pp. 1-5.

365 [16] J. D. Scargle, Studies in astronomical time series analysis. ii-statistical aspects of spectral analysis of unevenly spaced data, The Astrophysical Journal 263 (1982) $835-853$.

[17] M. S. Bartlett, Periodogram analysis and continuous spectra, Biometrika (1950) 1-16.

[18] B. Murmann, Adc performance survey 1997-2014, in: http://www.stanford.edu/ murmann/adcsurvey.html.

[19] C.-H. Yang, D. Markovic, A 2.89 mw 50gops 16× 16 16-core mimo sphere decoder in 90nm cmos, in: ESSCIRC, 2009. ESSCIRC'09. Proceedings of, IEEE, 2009, pp. 344-347. 


\section{Biography}

Yves LOUËT got his Ph.D. degree in 2000 and his Research Habilitation (HDR) in 2010 from Rennes University, France. From 2000 to 2002, he worked with SIRADEL (Rennes) on radio propagation modeling. He has been working with SUPELEC since 2002 as Professor. His research activities concern signal processing for Cognitive Radio 38о systems and especially PAPR reduction for multicarrier systems.

Daniel Le Guennec received the Engineering Degree from Supélec (Ecole Supérieure d'Electricité) in June 1977. He was Professor at Supélec from September 1978 to September 2013. He worked on adaptive signal processing, particularly as applied to digital communications problems, Carrier Recovery in communications systems with 385 low signal to noise ratio and one application to a DVB-S2 professional receiver design.

Babar AZIZ received his PhD degree from Cergy-Pontoise University, France in 2011. His current research interests are spectrum Sensing and Signal identification and Blind Standards identification for Cognitive Radio (DVB-T, DVB-T2, LTE ).

Samba Traoré received his MSc degree from INSA (Institut National des Science 390 Appliquée) Rennes in 2011. He is PhD student at Supélec since 2011 and his research activities concern non-uniform sub-sampling, non-uniform spectrum sensing and green cognitive radio. 